\title{
From pituitary adenoma to pituitary neuroendocrine tumor (PitNET): an International Pituitary Pathology Club proposal
}

\author{
S L Asa1, O Casar-Borota2, P Chanson³, E Delgrange4, P Earls5, S Ezzat6, \\ A Grossman7, H Ikeda ${ }^{8}, \mathbf{N}$ Inoshita9, N Karavitaki'10, M Korbonits"1, E R Laws Jr'2, \\ M B Lopes ${ }^{13}$, N Maartens'14, I E McCutcheon'15, O Mete1, H Nishioka'6, G Raverot17, \\ F Roncaroli18, W Saeger ${ }^{19}$, L V Syro20, A Vasiljevic ${ }^{21}$, C Villa22, A Wierinckx ${ }^{23}$, \\ $\mathrm{J}$ Trouillas ${ }^{24}$, and the attendees of 14th Meeting of the International Pituitary \\ Pathology Club, Annecy, France, November 2016
}

\author{
1Department of Pathology and Endocrine Oncology Site Group, Princess Margaret Cancer Centre, \\ University Health Network, Department of Laboratory Medicine and Pathobiology, University of \\ Toronto, Toronto, Ontario, Canada \\ 2Department of Pathology, Uppsala University Hospital, Uppsala, Sweden \\ 3Service of Endocrinology and Reproductive Diseases, Bicêtre Hospital, Paris, France \\ ${ }^{4}$ Department of Medicine, University of Louvain, Mont-sur-Meuse, Belgium \\ 5Department of Anatomical Pathology, St Vincent's Hospital, Sydney, Australia \\ ${ }^{6}$ Department of Medicine and Endocrine Oncology Site Group, Princess Margaret Cancer Centre, \\ University Health Network and University of Toronto, Toronto, Ontario, Canada \\ ${ }^{7}$ Department of Endocrinology, University of Oxford, Oxford, UK \\ 8Research Institute for Pituitary Disease, Southern Tohoku General Hospital, Fukushima, Japan \\ ${ }^{9}$ Department of Pathology, Toranomon Hospital, Tokyo, Japan \\ ${ }^{10}$ Department of Endocrinology, Queen Elizabeth Hospital, University of Birmingham, Birmingham, UK \\ 11Division of Endocrinology, Queen Mary Hospital, Barts and the London School of Medicine, London, UK \\ 12Department of Neurosurgery, Harvard Medical School, Brigham \& Women's Hospital, Boston, Massachusetts, USA \\ ${ }^{13}$ Departments of Pathology and Neurological Surgery, University of Virginia, Charlottesville, Virginia, USA \\ ${ }^{14}$ Department of Neurosurgery, Royal Melbourne Hospital, The University of Melbourne, Melbourne, Australia \\ 15Department of Neurosurgery, UT MD Anderson Cancer Center, Houston, Texas, USA \\ ${ }^{16}$ Department of Neurosurgery, Toranomon Hospital, Tokyo, Japan \\ 17Department of Endocrinology, Groupement Hospitalier EST, Hospices Civils de Lyon, \\ University of Lyon, Lyon, France \\ ${ }^{18}$ Department of Neuropathology, Imperial College, London, UK \\ ${ }^{19}$ Institute of Neuropathology of the University of Hamburg, Hamburg, Germany \\ ${ }^{20}$ Department of Neurosurgery, Hospital Pablo Tobon Uribe, Medellin, Colombia \\ ${ }^{21}$ Department of Pathology, Groupement Hospitalier EST, Hospices Civils de Lyon, University of Lyon, Lyon, France \\ 22Department of Pathology, Hôpital Foch, Suresnes, France \\ 23INSERM U1052, Cancer Research Center of Lyon, University of Lyon, Lyon, France \\ 24Faculty of Medicine Lyon-Est, University of Lyon, Lyon, France
}

Correspondence should be addressed to S L Asa or J Trouillas Email sylvia.asa@uhn.ca or Jacqueline.trouillas@ univ-lyon1.fr
๑ 2017 Society for Endocrinology Printed in Great Britain
Published by Bioscientifica Ltd. 


\section{Abstract}

The classification of neoplasms of adenohypophysial cells is misleading because of the simplistic distinction between adenoma and carcinoma, based solely on metastatic spread and the poor reproducibility and predictive value of the definition of atypical adenomas based on the detection of mitoses or expression of Ki-67 or p53. In addition, the current classification of neoplasms of the anterior pituitary does not accurately reflect the clinical spectrum of behavior. Invasion and regrowth of proliferative lesions and persistence of hormone hypersecretion cause significant morbidity and mortality. We propose a new terminology, pituitary neuroendocrine tumor (PitNET), which is consistent with that used for other neuroendocrine neoplasms and which recognizes the highly variable impact of these tumors on patients.

\section{Key Words \\ - pituitary tumors \\ - nomenclature}

Since the early work of Minkowski, who attributed acromegaly to a pituitary tumor (Minkowski 1887), neoplasms composed of pituitary adenohypophysial cells have been recognized as the cause of significant illness. However, Harvey Cushing attributed the term 'adenoma' even to patients who died of their disease (Cushing 1932).

We now recognize that these neoplasms are complex and heterogeneous; they present multiple clinical manifestations, including a wide range of proliferative and invasive behaviors (Asa 2011). Some are slowly growing small lesions that are clinically insignificant, some are small or large hormonally active lactotroph tumors that respond to medical therapy with shrinkage and reduced hormone secretion, whereas other small and minimally proliferative lesions cause the severe metabolic dysfunction of Cushing's disease or acromegaly. Many are large and invasive neoplasms that cause significant morbidity due to mass effects, with or without hormone excess syndromes. Traditional classifications only recognize malignancy, denoted as pituitary carcinoma, when there is evidence of distant metastasis or cerebrospinal spread (DeLellis et al. 2004). The attempt to classify a subgroup as 'atypical adenomas' based on the detection of mitoses or expression of Ki-67 or p53 (DeLellis et al. 2004) has proven to lack reproducibility and does not accurately predict recurrence or resistance to medical therapy (Miermeister et al. 2015). Recently, a clinicopathological classification with five grades identified Grade $2 \mathrm{~b}$ tumors as those with a high risk of recurrence or progression (Trouillas et al. 2013, Raverot et al. 2015). However, prediction of clinically aggressive behavior of these neoplasms, which occurs in approximately $10 \%$ of these tumors, remains debatable (Asa \& Ezzat 2016). The term 'adenoma', which defines a tumor as benign, does not seem appropriate to define aggressive and invasive pituitary tumors that cannot be resected and are refractory to therapy.

Patients and health care providers have long expressed frustration that these lesions are considered rare, benign and inconsequential. In most jurisdictions, they are not reported in cancer registries. Pituitary patients are often denied access to and/or health insurance coverage for therapies that would be provided for 'cancers'.

The International Pituitary Pathology Club, created in 1981, is a group of expert pathologists, endocrinologists, neurosurgeons and scientists who meet on a regular basis to discuss challenges and advances in the pituitary field. At the14th meeting in Annecy, France in November 2016, the subject of classification of these lesions was, as usual, controversial. However, there was consensus on one important aspect: pituitary endocrine neoplasms exhibit a spectrum of behaviors that are not entirely benign and can cause significant morbidity, even when they are not metastatic.

We therefore propose a reclassification of these tumors to apply terminology that has been widely accepted in other neuroendocrine tumors (NETs) (DeLellis et al. 2004). Pituitary hormone-producing cells are members of the family of neuroendocrine cells, similar to those of pancreatic islets, as well as dispersed endocrine cells of the gastrointestinal and respiratory tracts. Over the last two decades, there have been terminology shifts that reflect the potential for malignant behavior of even the most bland of those neuroendocrine neoplasms. They evolved from 'adenoma' to 'tumor' to recognize the lack of predictability. We therefore propose that neoplasms of adenohypophysial cells be termed 'pituitary neuroendocrine tumors'. 
Like other neuroendocrine neoplasms, many primary adenohypophysial tumors are indolent; they may be controlled by long-term pharmacologic treatment (e.g. dopamine-agonist therapy in the case of lactotroph tumors) or are non-invasive and cured by surgery. However, a variable proportion may recur despite remission. In addition, a large proportion (40\%) is invasive into the cavernous or sphenoid sinuses or cranial bones and cannot be totally removed by surgery. Moreover, some are resistant to the multiple medical treatments available and are considered to be clinically 'aggressive'. The features distinguishing these behaviors are not entirely clear at this time, and there is still no consensus on this matter. Moreover, there is no biomarker that can reliably predict malignancy as defined by metastatic spread. There is evidence that some morphologic subtypes of pituitary neoplasms of the various cell lineages exhibit more aggressive behavior (Asa \& Ezzat 2016). It is also clear that invasive lesions, which cannot be surgically resected are likely to result in considerable morbidity (Raverot et al. 2015); some may require radiotherapy or are rapidly proliferative despite surgery, radiotherapy and/or medical therapy, requiring chemotherapy, for example temozolomide or molecular targeted therapies. Accordingly, indications for novel therapies require elucidation of biomarkers that can guide personalized strategies.

This revision of nomenclature is not intended to negate the classification by morphologic cell type, but instead is intended to change the classification to 'tumor' rather than 'adenoma'; for example, a 'sparsely granulated somatotroph tumor' rather than 'sparsely granulated somatotroph adenoma'. The classification by morphologic cell type has been adopted by the World Health Organization (WHO) (DeLellis et al. 2004) and will remain in the next WHO classification that is underway. Although the new terminology of 'tumor' replacing 'adenoma' will not be incorporated in the 2017 WHO book, this change, as with previous terminologies that transitioned to 'NETs', will be gradually adopted to be included in the next edition. We hope that clarification of additional appropriate biomarkers will ultimately allow further refinement of the classification of pituitary neuroendocrine tumors.

The authors of this statement feel strongly that the time has come to reclassify clonal adenohypophysial proliferations under the umbrella of 'pituitary neuroendocrine tumor' (PitNET), a term that emphasizes the biological spectrum of these common endocrine neoplasms. Importantly, by analogy with other NETs, we encourage tumor registries to capture data on these tumors, thereby assisting efforts to clarify clinical and pathological features that can appropriately guide patient management. Pituitary neuroendocrine tumors are not simply endocrine diseases, but should be considered as tumors with endocrine manifestations within the context of oncology.

\section{Declaration of interest}

The authors declare that there is no conflict of interest that could be perceived as prejudicing the impartiality of this commentary.

\section{Funding}

This work did not receive any specific grant from any funding agency in the public, commercial, or not-for-profit sector

\section{Acknowledgement}

The authors acknowledge the participation and wise counsel of Prof. Günter Klöppel in the discussions that led to this manuscript. Attendees of the 14th Meeting of the International Pituitary Pathology Club, Annecy, France, November 2016 were: O Capraru, University of Medicine and Pharmacy, Tirgu Mures, Romania; L Chinezu, Department of Histology, University of Medicine and Pharmacy, Tirgu Mures, Romania; H Dufour, Department of Neurosurgery, La Timone Hospital, Marseille, France; N Fukuhara, Department of Hypothalamic and Pituitary Surgery, Toranomon Hospital, Tokyo, Japan; S Gaillard, Department of Surgery, Hôpital Foch, Suresnes, France; F Guaraldi, Division of Endocrinology, University of Turin, Turin, Italy; P E Harris, IPSEN, Paris, France; M L JaffrainRea, University of L'Aquila, Coppito, L'Aquila, Italy; E Jouanneau, Department of Neurosurgery, Groupement Hospitalier EST, Hospices Civils de Lyon, University of Lyon, Lyon, France; I Kraljevic, Department of Endocrinology, University Center, Zagreb, Croatia; E Manojlović-Gačić, Institute of Pathology, Medical Faculty, University of Belgrade, Belgrade, Serbia; O Tachibana, Department of Neurosurgery, Kanazawa Medical University, Ishikawa, Japan; and M Theodoropoulou, Ludwig Maximilians Universität München, Munich, Germany.

\section{References}

Asa SL 2011 In Tumors of the Pituitary Gland. AFIP Atlas of Tumor Pathology, Series 4, Fascicle 15. Ed SG Silverberg. Silver Spring, MD, USA: ARP Press.

Asa SL \& Ezzat S 2016 Aggressive pituitary tumors or localized pituitary carcinomas: defining pituitary tumors. Expert Review of Endocrinology and Metabolism 11 149-162. (doi:10.1586/17446651.2016.1153422)

Cushing H 1932 The basophil adenomas of the pituitary body and their clinical manifestations (pituitary basophilism). Bulletin of the Johns Hopkins Hospital 50 137-195.

DeLellis RA, Lloyd RV, Heitz PU \& Eng C 2004 Pathology and Genetics of Tumours of Endocrine Organs. Lyons, France: IARC Press.

Miermeister CP, Petersenn S, Buchfelder M, Fahlbusch R, Ludecke DK, Holsken A, Bergmann M, Knappe HU, Hans VH, Flitsch J, et al. 2015 Histological criteria for atypical pituitary adenomas - data from the German pituitary adenoma registry suggests modifications. Acta Neuropathologica Communications 3 50. (doi:10.1186/s40478-0150229-8)
(C) 2017 Society for Endocrinology Printed in Great Britain
Published by Bioscientifica Ltd 
Minkowski O 1887 Ueber einen fall von akromegalie. Klinische Wochenschrift 24 371-374.

Raverot G, Vasiljevic A, Jouanneau E \& Trouillas J 2015 A prognostic clinicopathologic classification of pituitary endocrine tumors. Endocrinology Metabolism Clinics of North America 44 11-18. (doi:10.1016/j.ecl.2014.10.001)
Trouillas J, Roy P, Sturm N, Dantony E, Cortet-Rudelli C, Viennet G, Bonneville JF, Assaker R, Auger C, Brue T, et al. 2013 A new prognostic clinicopathological classification of pituitary adenomas: a multicentric case-control study of 410 patients with 8 years postoperative follow-up. Acta Neuropathologica 126 123-135. (doi:10.1007/s00401-013-1084-y)

Received in final form 31 January 2017

Accepted 1 February 2017 